\title{
Job Insecurity, Work-Family Imbalance, and Hostile Work Environment: Prevalence Data from the 2010 National Health Interview Survey
}

\author{
Toni Alterman, $\mathrm{PhD},{ }^{1}{ }^{*}$ Sara E. Luckhaupt, MD, $\mathrm{MPH}^{1}{ }^{1}$ James $\mathrm{M}$. Dahlhamer, $\mathrm{PhD}^{2}{ }^{2}$ \\ Brian W. Ward, $\mathrm{PhD}^{2}$ and Geoffrey M. Calvert, $\mathrm{MD}, \mathrm{MPH}^{1}$
}

\begin{abstract}
Background Little nationally representative information on job insecurity, workfamily imbalance, and hostile work environments experienced by workers in the US is available.

Methods Prevalence rates from the 2010 National Health Interview Survey (NHIS) were calculated for three workplace psychosocial factors (job insecurity, work-family imbalance, bullying/harassment) using SUDAAN to account for the complex NHIS sample design.

Results Data were available for 17,524 adults who worked in the 12 months that preceded the interview. Overall prevalence rates were $31.7 \%$ for job insecurity, $16.3 \%$ for work-family imbalance, and $7.8 \%$ for hostile work environment (being bullied or harassed). The highest prevalence rate of job insecurity was found for construction and extraction occupations. Workers in legal occupations had the highest prevalence rate of work-family imbalance. Workers in protective service occupations had the highest prevalence rate of hostile work environment.

Conclusions We identified demographic characteristics along with industries and occupations with the highest prevalence rates for three adverse workplace psychosocial factors. These data can be used for benchmarking and identification of targets for investigation and intervention activities. Am. J. Ind. Med. 56:660-669, 2013.
\end{abstract}

(C) 2012 Wiley Periodicals, Inc.

KEY WORDS: job stress; national survey; bullying; job insecurity; work-family imbalance

1 Division of Surveillance, Hazard Evaluations and Field Studies, National Institute for Occupational Safety and Health, Centers for Disease Control and Prevention, Cincinnati, Ohio

${ }^{2}$ Division of Health Interview Statistics, National Center for Health Statistics, Centers for

Disease Control and Prevention, Hyattsville, Maryland

Contract grant sponsor: NHIS.

Contract grant sponsor: US Government.

Disclaimer: The findings and conclusions in this report are those of the authors and do not necessarily represent the views of the National Institute for Occupational Safety and Health, nor the National Center for Health Statistics.

Competing interest: None declared.

${ }^{*}$ Correspondence to: Dr. Toni Alterman, PhD, Division of Surveillance, Hazard Evaluations and Field Studies, National Institute for Occupational Safety and Health, 4676 Columbia Parkway, R-17, Cincinnati, OH 45226.E-mail: talterman@ @cdc.gov

Accepted 31 August 2012

DOl 10.1002/ajim.22123. Published online 28 September 2012 in Wiley Online Library (wileyonlinelibrary.com).

\section{INTRODUCTION}

Little nationally representative information on job insecurity, work-family imbalance, and hostile work environments experienced by US workers are available. In addition, "National surveillance of psychosocial risk factors in the workplace is important to record the changing work environment and for the development (and monitoring) of policies and programs to prevent stress and promote mental and physical health and well-being at work" [Dollard et al., 2007]. Prevalence rates for workplace psychosocial risk factors can provide data for benchmarking 
and identification of targets for investigation and intervention activities.

Questions on exposures to three workplace psychosocial factors (job insecurity, work-family imbalance, and bullying or harassment) were developed after literature reviews and consultation with experts in the field of occupational health and work stress for inclusion in the National Institute for Occupational Safety and Health (NIOSH) sponsored Occupational Health Supplement (OHS) to the 2010 National Health Interview Survey (NHIS). This article presents national self-reported prevalence rates of these workplace psychosocial exposures. Data were collected from a nationally representative sample of adults who reported working at the time of the interview, or who had worked in the previous 12 months. Although, a comprehensive analysis of the association between these workplace psychosocial factors and health is beyond the scope of a single article, data are available in a public use dataset (http://www.cdc.gov/nchs/nhis/nhis_2010_ data_release.htm) and we encourage researchers to analyze the data further. In this article, we provide population prevalence rates for three workplace psychosocial factors by demographic characteristics, industry and occupation. We also provide age, sex, and race/ethnicity adjusted prevalence rates of workplace psychosocial factors by industry and occupation so that researchers may use these for imputation of job characteristics in their own analyses when exposure data are lacking.

\section{METHODS}

Data from the 2010, NHIS were used for this study. The NHIS is an annual, multi-purpose health survey, and the principal source of information about the health of the civilian, non-institutionalized, household population of the United States. The survey is conducted by the National Center for Health Statistics (NCHS), Centers for Disease Control and Prevention, and utilizes a multi-stage, clustered sample design, with over sampling of black, Hispanic, and Asian persons. Black, Hispanic, and Asian adults aged 65 or older are also over sampled to complete the sample adult module.

Interviewers with the US Census Bureau administer in-person interviews (some telephone follow-up is allowed) using computer assisted personal interviewing (CAPI). The survey instrument contains four main modules: Household, Family, Sample Child, and Sample Adult. A household respondent provides demographic information on all members of the household in the household composition module. For each family within a household, the family module is completed by one family respondent who provides sociodemographic and health information on all members of the family. Additional health information is collected from one randomly selected adult (sample adult) aged 18 years or over, and from the parent or guardian of one randomly selected child under age 18 (if there are children in the family). Occupational Health Supplement (OHS) questions were imbedded into the Sample Adult questionnaire. In 2010, NHIS interviews were conducted in 34,329 households, accounting for 89,976 persons in 35,177 families. Detailed data were collected from 27,157 sample adults. The household response rate was $79.5 \%$, the conditional sample adult response rate was $77.3 \%$ and the final sample adult response rate was $60.8 \%$ [Division of Health Interview Statistics, 2010]. Survey questions were developed after consultation with content experts and thorough literature reviews.

In 2010, National Health Interview Survey (NHIS) was approved by the Research Ethics Review Board of the National Center for Health Statistics (Protocol \#2009-16) and the US Office of Management and Budget (Control \#0920-0214). Written consent for participation in the 2010 NHIS was not received, but instead all 2010 NHIS respondents provided oral consent prior to participation.

\section{Study Definitions}

A total of 17,524 currently or recently employed sample adults were included in this study. Of these, 15,649 $(89.3 \%)$ had a job during the week prior to the survey, and $1,875(10.7 \%)$ did not have a job in the prior week but did work in the 12 months preceding the interview. We present national prevalence rates for job insecurity and work-family imbalance for the current main job held by currently employed sample adults. We present national prevalence rates for hostile work environment (being bullied or harassed) for the current main job held by currently employed sample adults and for most recent job held by sample adults not working at the time of interview, but who worked at some time in the previous 12 months.

Employment was defined as working for pay at a job or business or working, but not for pay, at a family-owned business or farm. To ensure that respondents answered about the job of interest, questions and question sets often used a lead-in similar to the following: "The next few questions refer to [fill: your job as a (JOB DESCRIPTION) with (EMPLOYER NAME)/your current, MAIN job/the job you held [most recently]." We then classified current/recent workers by demographic (sex, age, race/ ethnicity, marital status, education, and class of worker) and geographic characteristics (place of residence and region). Geographic classification was based on the location of the respondent's home as within or outside a metropolitan statistical area (MSA). Analysis by educational status was limited to workers aged 25 years and older. Industry and occupation categories were created by NCHS based on the North American Industry Classification System 
(NAICS) and the Standard Occupational Classification System (SOC) codes.

\section{Psychosocial Factors}

Job insecurity and work family imbalance were measured by the following questions: "Please tell me whether you: strongly agree, agree, disagree, or strongly disagree with each of these statements." "I am worried about becoming unemployed" and "It is easy for me to combine work with family responsibilities," respectively. Responses of "strongly agree" and "agree" to the first statement were defined as job insecurity for this analysis, while responses of "strongly disagree" and "disagree" to the second statement were defined as work-family imbalance. Hostile work environment was defined as those answering "yes" to the question "DURING THE PAST 12 MONTHS were you threatened, bullied, or harassed by anyone while you were on the job?"

\section{Analysis}

All analyses were conducted using SAS-callable SUDAAN software version 10.0 [RTI, 2008] to account for the complex sampling design of the NHIS. To represent the US civilian, non-institutionalized population age 18 years and over, and to estimate the total number of employed US civilian workers represented by each individual in the sample, all estimates were weighted using the NHIS sample adult record weight. Point estimates with a relative standard error (RSE) greater than $30 \%$ but less than or equal to $50 \%$ are noted in the text and indicated with an "**" in the tables as they do not meet the NCHS standards of reliability/precision. Estimates with a RSE greater than $50 \%$ or based on cell sizes less than 10 cases are not shown.

In order to assess patterns of prevalence for psychosocial exposures among workers by industry and occupation group, we ranked groups from highest to lowest unadjusted prevalence rate. Note that these rankings do not account for whether or not the differences between estimates were statistically significant. However, we did calculate significance tests that tested for statistically significant differences between the industry and occupation groups with the highest prevalence rates for psychosocial exposures, and the prevalence rate of these exposures for all workers combined. These significance tests were adjusted such that the estimated standard error of the difference between prevalence rates for industry and occupation groups and all workers accounted for non-independence of industry and occupation groups and all workers by incorporating their covariance [a method used in Cohen and Makuc, 2008]. Differences that were statistically significant $(P<0.05)$ for select variables are noted in the text. Tetrachoric correlations between psychosocial factors are provided in Appendix A.

When examining the prevalence rate of psychosocial factors among various industry and occupation groups, we present unadjusted prevalence rates that may be useful for comparisons to unadjusted data from other sources (e.g., O*NET), and for identifying groups of workers with the higher burdens of exposure to target with preventive strategies. Some researchers may prefer to use adjusted prevalence rates for industry and occupation groups to make our estimates comparable to those of the Quality of Employment Surveys, which were adjusted for age, sex and race [Karasek et al., 1988; Schwartz et al., 1988; Pieper et al., 1989; Reed et al., 1989; Alterman et al., 1994]. In Table I we present prevalence rates adjusted by age, sex, and race/ethnicity using the projected 2000 US population as the standard population [Day, 1996]. Although, we do not discuss individual adjusted prevalence rates in this article due to space limitations, we are making them available for researchers to use.

\section{RESULTS}

Employment status data were available for 27,157 sample adults in the 2010 NHIS, who represent approximately 229 million civilian non-institutionalized US adults (Table II). The sample included 17,524 adults (weighted proportion $=67.7 \%$ ) who were employed in the past 12 months (current/recent workers); 7,915 (26.7\%) who were not employed in the past 12 months, but were employed at some time in the past (former workers); and $1,704(5.7 \%)$ who were never employed.

\section{Psychosocial Factors}

\section{Job insecurity}

The overall prevalence rate of current workers reporting job insecurity (i.e., worry about becoming unemployed) was $31.7 \%$ (Table II). Job insecurity was higher for men $(33.0 \%)$ than women $(30.2 \% ; P<0.01)$; workers aged 30-44 (34.3\%) and 45-64 (34.1\%) compared with other age groups $(P<0.01$ for all pair-wise comparisons); Hispanic workers $(47.2 \%)$ compared with other racial/ethnic groups $(P<0.01$ for all pair-wise comparisons), with the exception of non-Hispanic American Indian/Alaska Native workers; divorced or separated workers $(39.5 \%)$ compared with other relationship statuses $(P<0.01$ for all pair-wise comparisons); and workers having less than a high school diploma (48.6\%) compared to workers having more education $(P<0.01$ for all pair-wise comparisons). Prevalence rates of job insecurity were also higher for 
TABLE I. Population-Based Prevalence Rates and Confidence Intervals for Psychosocial Exposures Among US Working Adults, by Industry and Occupation, Adjusted for Age, Sex, and Race/Ethnicity (National Health Interview Survey, 2010)

Adj. \%(95\% Cl)

\begin{tabular}{|c|c|c|c|}
\hline & \multicolumn{3}{|c|}{ Adj. \% (95\% Cl) } \\
\hline & Job insecurity ${ }^{a}$ & Work-family imbalance ${ }^{a}$ & Hostile work environment $^{\mathrm{b}}$ \\
\hline \multicolumn{4}{|l|}{ Industry } \\
\hline Agriculture, forestry,fishing, and hunting & $17.4(12.8-23.3)$ & $10.4(6.6-16.1)$ & $\dagger$ \\
\hline Mining & $38.8(30.7-7.5)$ & $22.2(16.5-9.3)$ & $\dagger$ \\
\hline Utilities & $18.4(11.7-27.7)$ & $17.2(11.4-25.1)$ & $* 5.0(2.7-9.1)$ \\
\hline Construction & $41.3(37.0-45.8)$ & $14.2(11.3-17.7)$ & $5.1(3.1-8.1)$ \\
\hline Manufacturing & $31.2(28.5-34.0)$ & $16.5(14.2-19.0)$ & $5.5(4.0-7.5)$ \\
\hline Wholesale trade & $26.0(21.4-31.2)$ & $12.5(9.6-16.1)$ & $5.5(3.3-8.9)$ \\
\hline Retail trade & $30.7(28.0-33.5)$ & $16.0(13.9-18.4)$ & $10.2(8.6-12.1)$ \\
\hline Transportation and warehousing & $28.3(24.2-32.8)$ & $16.9(11.9-23.5)$ & $9.4(7.2-12.2)$ \\
\hline Information & $41.4(35.2-47.8)$ & $15.6(11.9-20.2)$ & $7.0(4.7-10.4)$ \\
\hline Finance and insurance & $25.6(21.6-30.1)$ & $12.2(10.0-14.8)$ & $5.1(3.1-8.3)$ \\
\hline Real Estate and rental and leasing & $29.6(24.0-35.8)$ & $14.2(10.4-19.2)$ & $7.7(4.9-12.0)$ \\
\hline Professional, scientific, and technical services & $25.6(22.6-28.8)$ & $15.8(13.2-18.7)$ & $5.4(4.1-7.0)$ \\
\hline Management of companies and enterprises & $\dagger$ & $\dagger$ & $\dagger$ \\
\hline Administrative and support, and waste management and remediation services & $33.4(28.5-38.7)$ & $14.2(11.5-17.5)$ & $7.2(5.2-9.8)$ \\
\hline Educationservices & $26.3(23.2-29.7)$ & $16.9(13.9-20.3)$ & $8.1(6.6-10.1)$ \\
\hline Health care and social assistance & $22.3(19.4-25.6)$ & $13.6(11.4-16.2)$ & $7.2(5.6-9.2)$ \\
\hline Arts, entertainment, and recreation & $30.8(25.5-36.7)$ & $13.1(9.8-17.2)$ & $6.7(4.6-9.7)$ \\
\hline Accommodation and food services & $28.2(24.9-31.7)$ & $17.8(15.2-20.7)$ & $7.2(5.5-9.2)$ \\
\hline Other services (except public administration) & $25.4(22.2-28.9)$ & $11.9(9.6-14.8)$ & $5.3(3.7-7.4)$ \\
\hline Public administration & $18.4(15.3-21.8)$ & $13.5(11.2-16.1)$ & 14.2(11.6-17.2) \\
\hline \multicolumn{4}{|l|}{ Occupation } \\
\hline Management & $22.1(19.6-24.7)$ & $16.3(14.3-18.5)$ & $5.8(4.6-7.3)$ \\
\hline Business and financial operations & $25.8(21.8-30.2)$ & 13.3(11.2-15.8) & $4.6(3.1-6.7)$ \\
\hline Computer and mathematical & $28.3(21.5-36.3)$ & $12.0(9.0-16.0)$ & $3.7(2.2-6.4)$ \\
\hline Architecture and engineering & $26.6(21.1-33.1)$ & $10.3(6.7-15.6)$ & $* 4.2(2.2-8.2)$ \\
\hline Life, physical, and social science & $20.1(15.0-26.5)$ & $16.6(12.0-22.5)$ & $\dagger$ \\
\hline Community and social services & $26.8(21.5-32.8)$ & $13.0(9.6-17.4)$ & $13.5(9.7-18.3)$ \\
\hline Legal & $29.0(20.9-38.8)$ & $20.9(15.4-27.7)$ & $6.8(3.8-11.9)$ \\
\hline Education, training, and library & $24.6(21.5-27.9)$ & $16.0(13.4-19.0)$ & $8.1(6.0-10.9)$ \\
\hline Arts, design, entertainment, sports and media & $26.6(22.3-31.3)$ & $12.1(9.0-16.0)$ & $6.6(4.4-9.6)$ \\
\hline Healthcare practitioners and technical & $15.9(12.9-19.6)$ & $16.4(13.0-20.5)$ & $6.8(5.3-8.8)$ \\
\hline Healthcare support & $37.9(32.0-44.3)$ & $15.2(10.0-22.4)$ & $12.2(7.8-18.7)$ \\
\hline Protective service & $20.7(16.3-26.0)$ & $23.3(16.4-32.0)$ & $24.2(19.1-30.2)$ \\
\hline Food preparation and serving related & $31.0(27.1-35.2)$ & $16.0(13.2-19.3)$ & $7.9(5.8-10.7)$ \\
\hline Building and grounds cleaning and maintenance & $30.3(25.9-35.1)$ & $10.9(8.1-14.4)$ & $7.0(4.5-10.8)$ \\
\hline Personal care and service & $21.6(17.5-26.4)$ & $17.2(12.5-23.2)$ & $7.5(4.8-11.8)$ \\
\hline Sales and related & $30.8(28.2-33.6)$ & $16.3(14.1-18.6)$ & $8.3(6.9-9.9)$ \\
\hline Office and administrative support & $28.2(25.6-30.9)$ & $11.7(9.9-13.8)$ & $8.2(6.7-9.9)$ \\
\hline Farming, fishing, and forestry & $16.6(11.4-23.5)$ & $8.1(5.0-12.7)$ & $* 0.6(0.3-1.2)$ \\
\hline Construction and extraction & $43.8(36.0-51.9)$ & $17.8(11.8-26.1)$ & $5.7(3.5-9.2)$ \\
\hline Installation, maintenance, and repair & $34.2(27.8-41.3)$ & $18.4(13.4-24.7)$ & $10.7(6.8-16.6)$ \\
\hline Production & $36.5(32.6-40.5)$ & $15.6(12.7-19.0)$ & $5.8(4.3-7.8)$ \\
\hline Transportation and material moving & $30.3(26.2-34.8)$ & $14.7(12.2-17.5)$ & $9.1(7.0-11.7)$ \\
\hline
\end{tabular}

Adj., adjusted; $\mathrm{Cl}$, confidence interval.

All estimates are weighted. Estimates adjusted by age, sex, and race/ethnicity using the projected 2000 US population as the standard population.

ancludes currently employed adults.

bIncludes currently employed adults, and adults not currently employed but employed at some time in the past 12 months.

*Estimates preceded by an asterisk have a relative standard error $>30 \%$ and $\leq 50 \%$ and should be used with caution as they do not meet standards of reliability/precision.

${ }^{\dagger}$ Estimates with a relative standard error $>50 \%$ or based on cell sizes $\leq 10$ are not shown as they do not meet standards of reliability/precision. 
TABLE II. Population-Based Prevalence Rates and Confidence Intervals of Psychosocial Exposures Among Working US Adults, by Demographic and Geographic Characteristics (National Health Interview Survey, 2010)

\begin{tabular}{|c|c|c|c|c|c|c|c|c|}
\hline & \multirow[b]{2}{*}{ Sample $^{a}$} & \multirow[b]{2}{*}{$\begin{array}{l}\text { Est. population } \\
\text { in thousands }\end{array}$} & \multicolumn{2}{|c|}{ Job insecurity ${ }^{b}$} & \multicolumn{2}{|c|}{ Work-family imbalance ${ }^{b}$} & \multicolumn{2}{|c|}{ Hostile work environment ${ }^{c}$} \\
\hline & & & Exp. $^{a}$ & $\begin{array}{c}(95 \% \mathrm{CI}) \\
(\%)\end{array}$ & Exp. $^{a}$ & $\begin{array}{c}(95 \% \mathrm{CI}) \\
(\%)\end{array}$ & Exp. $^{a}$ & $\begin{array}{c}(95 \% \mathrm{CI}) \\
(\%)\end{array}$ \\
\hline Total & 17,524 & $1,55,262$ & 5,233 & $31.7(30.8-32.6)$ & 2,589 & $16.3(15.6-17.1)$ & 1,422 & $7.8(7.4-8.4)$ \\
\hline \multicolumn{9}{|l|}{ Sex } \\
\hline Male & 8,500 & 81,412 & 2,628 & $33.0(31.7-34.4)$ & 1,206 & $15.7(14.7-16.7)$ & 575 & $6.5(5.9-7.1)$ \\
\hline Female & 9,024 & 73,850 & 2,605 & $30.2(29.0-31.4)$ & 1,383 & $17.1(16.1-18.1)$ & 847 & $9.3(8.6-10.1)$ \\
\hline \multicolumn{9}{|l|}{ Age group (yrs.) } \\
\hline $18-29$ & 4,059 & 38,916 & 1,008 & $27.5(25.7-29.4)$ & 538 & $15.3(13.9-16.8)$ & 316 & $7.9(6.9-9.0)$ \\
\hline $30-44$ & 5,967 & 49,624 & 1,983 & $34.3(32.7-35.8)$ & 1,053 & $18.8(17.5-20.0)$ & 475 & $7.7(6.9-8.5)$ \\
\hline $45-64$ & 6,506 & 59,041 & 2,123 & $34.1(32.7-35.6)$ & 950 & $16.1(14.9-17.2)$ & 596 & $8.5(7.7-9.4)$ \\
\hline$\geq 65$ & 992 & 7,681 & 119 & $13.5(11.1-16.4)$ & 48 & $6.3(4.5-8.7)$ & 35 & $3.4(2.4-4.9)$ \\
\hline \multicolumn{9}{|l|}{ Race/ethnicity } \\
\hline Non-Hispanic white & 9,997 & $1,06,033$ & 2,509 & $27.9(26.8-29.0)$ & 1,406 & $16.1(15.2-17.1)$ & 812 & $7.9(7.3-8.5)$ \\
\hline Non-Hispanic black & 2,600 & 16,822 & 798 & $34.7(32.2-37.4)$ & 441 & $19.3(17.4-21.4)$ & 233 & $8.2(7.0-9.5)$ \\
\hline Hispanic & 3,464 & 22,273 & 1,472 & $47.2(45.0-49.5)$ & 522 & $15.3(13.9-16.9)$ & 262 & $7.4(6.4-8.5)$ \\
\hline \multicolumn{9}{|l|}{ Non-Hispanic Asian/Native } \\
\hline Hawaiian or Other Pacific Islander & 1,132 & 7,450 & 368 & $34.0(30.6-37.7)$ & 172 & $16.7(14.1-19.8)$ & 62 & $5.1(3.8-6.7)$ \\
\hline \multicolumn{9}{|l|}{ Non-Hispanic American } \\
\hline Indian/Alaska Native & 69 & 764 & 15 & $* 25.8(12.8-45.1)$ & $\dagger$ & $\dagger$ & 11 & $*_{11.6}(5.6-22.5)$ \\
\hline Non-Hispanic Other race & 262 & 1,920 & 71 & $30.4(23.4-38.3)$ & 38 & $15.7(10.8-22.2)$ & 42 & $16.9(11.9-23.3)$ \\
\hline \multicolumn{9}{|l|}{ Marital status } \\
\hline Married & 8,105 & 86,431 & 2,331 & $30.5(29.3-31.7)$ & 1,208 & $16.4(15.4-17.4)$ & 548 & $6.7(6.1-7.4)$ \\
\hline Widowed & 514 & 2,902 & 120 & $25.6(21.2-30.5)$ & 54 & $13.3(9.5-18.2)$ & 44 & $8.3(6.0-11.4)$ \\
\hline Divorced or separated & 2,983 & 17,626 & 1,056 & $39.5(37.3-41.8)$ & 497 & $18.6(16.8-20.5)$ & 337 & $11.9(10.5-13.4)$ \\
\hline Never married & 4,661 & 35,565 & 1,356 & $31.5(29.6-33.4)$ & 645 & $15.4(14.0-16.8)$ & 369 & $7.8(6.8-8.9)$ \\
\hline Living with partner & 1,232 & 12,564 & 363 & $31.3(28.4-34.5)$ & 180 & $16.3(13.9-19.0)$ & 120 & $10.2(8.4-12.2)$ \\
\hline \multicolumn{9}{|l|}{ Education } \\
\hline Less than HS diploma & 1,812 & 13,049 & 770 & $48.6(45.5-51.8)$ & 246 & $14.8(12.9-17.0)$ & 114 & $6.3(5.0-7.9)$ \\
\hline HS/GED diploma & 3,685 & 32,164 & 1,266 & $36.7(34.8-38.7)$ & 554 & $15.8(14.4-17.4)$ & 332 & $8.7(7.7-9.8)$ \\
\hline Somecollege & 4,656 & 39,755 & 1,409 & $32.5(30.8-34.2)$ & 695 & $16.7(15.4-18.2)$ & 416 & $8.4(7.5-9.4)$ \\
\hline $\mathrm{BA} / \mathrm{BS}$ degree and higher & 5,284 & 48,309 & 1,346 & $26.3(24.8-27.7)$ & 863 & $18.0(16.7-19.3)$ & 420 & $7.7(6.9-8.6)$ \\
\hline \multicolumn{9}{|l|}{ Class of worker } \\
\hline Private company for wages & 12,859 & $1,13,927$ & 3,968 & $32.9(31.9-34.0)$ & 1,901 & $16.4(15.6-17.3)$ & 989 & $7.4(6.8-8.0)$ \\
\hline Federal, state, or local government & 2,915 & 25,494 & 697 & $25.4(23.4-27.5)$ & 443 & $17.1(15.4-18.9)$ & 349 & $11.8(10.5-13.3)$ \\
\hline Self-employed in own business, professional or farm & 1,594 & 14,520 & 530 & $33.9(31.1-36.9)$ & 230 & $15.2(13.0-17.6)$ & 72 & $4.6(3.5-6.0)$ \\
\hline Working without pay in family owned business or farm & 78 & 718 & 19 & $22.8(13.6-35.6)$ & $\dagger$ & + & $\dagger$ & $\dagger$ \\
\hline \multicolumn{9}{|l|}{ Place of residence } \\
\hline Large MSA & 9,796 & 84,107 & 3,264 & $34.9(33.7-36.2)$ & 1,553 & $17.4(16.4-18.5)$ & 770 & $7.6(6.9-8.3)$ \\
\hline Small MSA & 5,266 & 48,741 & 1,376 & $28.5(26.9-30.1)$ & 704 & $14.8(13.6-16.0)$ & 454 & $8.3(7.5-9.3)$ \\
\hline Notin MSA & 2,462 & 22,414 & 593 & $26.6(24.4-28.8)$ & 332 & $15.6(13.8-17.7)$ & 198 & $7.8(6.6-9.3)$ \\
\hline \multicolumn{9}{|l|}{ Region } \\
\hline Northeast & 2,685 & 27,043 & 828 & $32.1(29.8-34.4)$ & 459 & $19.2(17.1-21.4)$ & 213 & $8.0(6.9-9.3)$ \\
\hline Midwest & 3,948 & 36,932 & 1,003 & $27.8(25.9-29.7)$ & 539 & $15.5(14.1-17.0)$ & 348 & $7.8(6.9-9.0)$ \\
\hline South & 6,421 & 54,415 & 1,910 & $31.1(29.7-32.6)$ & 931 & $15.4(14.3-16.6)$ & 509 & $7.5(6.8-8.3)$ \\
\hline West & 4,470 & 36,873 & 1,492 & $36.3(34.5-38.2)$ & 660 & $16.5(15.1-18.0)$ & 352 & $8.2(7.1-9.4)$ \\
\hline
\end{tabular}

Est., estimated; Exp., exposed; $\mathrm{Cl}$, confidence interval.

All estimates weighted unless otherwise noted.

aUnweighted.

${ }^{\mathrm{b}}$ Includes currently employed adults.

'Includes currently employed adults, and adults not currently employed but employed at some time in the past 12 months.

${ }^{*}$ Relative standard error (RSE) $>30 \% \geq 50 \%$ are noted in the tables as they do not meet the NCHS standards of reliability/precision.

${ }^{\dagger}$ Estimates with a relative standard error $>50 \%$ or based on cell sizes $<10$ are not shown as they do not meet standards of reliability/precision. 
workers who were self-employed in their own business, professional practice or farm (33.9\%), or working at a private company for wages $(32.9 \%)$ compared with adults working for Federal, state, or local government $(25.4 \%$; $P<0.01$ for both pair-wise comparisons).

Among industry groups (see Table III), workers in Construction (54.7\%; $P<0.01$ ), Information (45.1\%; $P<0.01$ ), and Administrative Support and Waste Management Companies $(39.9 \% ; P<0.01)$ had a higher prevalence rate of job insecurity compared to all employed adults $(31.7 \%)$. A lower prevalence rate of job insecurity was found among those in Utilities industries (17.7\%; $P<0.01)$. Among occupational groups, higher prevalence rates for job insecurity were observed for Construction and Extraction occupations $(55.7 \% ; P<0.01)$, followed by Building and Grounds Cleaning and Maintenance occupations (40.8\%; $P<0.01$ ), and Production occupations (39.8\%; $P<0.01)$. On the other hand, compared to the prevalence rate for all employed adults, workers in Healthcare Practitioner and Technical occupations (18.0\%; $P<0.01)$ had a lower prevalence rate of job insecurity.

\section{Work-Family Imbalance}

As seen in Table II, the overall prevalence rate of workers reporting difficulty balancing (i.e., an imbalance between) work and family was $16.3 \%$. Imbalance was more prevalent among workers aged 30-44 (18.8\%) compared with other age groups $(P<.01$ for all pair-wise comparisons); non-Hispanic black workers (19.3\%) compared with non-Hispanic white workers (16.1\%; $P<0.01$ ), and Hispanic workers $(15.3 \% ; P<0.01)$; divorced or separated workers $(18.6 \%)$ compared with married workers $(16.4 \% ; P<0.05)$, widowed workers (13.3\%; $P<0.05)$, and never married workers $(15.4 \%$; $P<0.01)$; and workers having a Bachelor's degree and higher $(18.0 \%)$ compared with workers having a high school diploma or G.E.D. $(15.8 \% ; P<0.05)$, and workers with less than a high school education (14.8\%; $P<0.05)$.

Workers in Agriculture, Forestry, Fishing, and Hunting industries $(9.4 \% ; P<0.01)$ had a lower prevalence rate of work-family imbalance (Table III) compared to all employed adults (16.3\%). Among occupations, a higher prevalence rate of work-family imbalance was found in Legal occupations $(26.1 \% ; P<0.05)$, whereas a lower prevalence rate was observed for workers in Office and Administrative Support $(13.7 \% ; P<0.01)$ and Farming, Forestry, and Fishing occupations $(9.5 \% ; P<0.01)$.

\section{Hostile Work Environment}

The national prevalence rate for workers reporting having been threatened, bullied, or harassed by anyone on the job (i.e., working in a hostile work environment) was $7.8 \%$ (Table II). Higher prevalence rates for experiencing a hostile work environment were identified for women $(9.3 \%)$ compared with men $(6.5 \% ; P<0.01)$; non-Hispanic other workers $(16.9 \%)$ compared to other racial/ethnic groups (excluding non-Hispanic American Indian/Alaska native adults; $P<0.01$ for all pair-wise comparisons); divorced or separated workers (11.9\%) compared to married workers $(6.7 \% ; P<0.01)$, widowed workers $(8.3 \% ; P<0.05)$, and never married workers $(7.8 \% ; P<0.01)$; and workers with only a high school diploma or GED $(8.7 \%)$ and workers with some college education (8.4\%) compared to workers with less than a high school education $(P<0.05$ for both pair-wise comparisons). Workers aged 65 and older $(3.4 \%)$ had a lower prevalence rate of working in a hostile work environment compared to workers in other age groups $(P<0.01$ for all pair-wise comparisons), while a higher prevalence rate of being threatened, bullied, or harassed by anyone on the job was identified for adults working for a Federal, state, or local government $(11.8 \%)$ compared to employees of private firms $(7.4 \% ; \quad P<0.01)$ and self-employed adults (4.6\%; $P<0.01)$.

Among industry groups, workers with higher prevalence rates of a hostile work environment (Table III), compared to all adults employed at some time in the past 12 months (7.8\%), were in Public Administration (16.2\%; $P<0.01)$ and Retail Trade industries $(10.1 \%$; $P<0.01)$. Lower prevalence rates of a hostile work environment were reported among those working in Construction $(5.0 \% ; P<0.01)$; Finance and Insurance (5.1\%; $P<0.01)$; Manufacturing (5.4\%; $P<0.01$ ); and Professional, Scientific, and Technical Services industries $(5.5 \% ; P<0.01)$. For occupational groups, workers in Protective Service reported a higher prevalence rate $(24.6 \% ; P<0.01)$ of hostile work environments compared to the prevalence rate for all adults employed at some time in the past 12 months. A similar finding emerged for workers in Community and Social Service occupations $(15.7 \% ; \quad P<0.01)$. Lower prevalence rates were observed among Architecture and Engineering (3.9\%; $P<0.01)$, Computer and Mathematical (4.4\%; $P<0.01)$, Business and Financial Operations $(4.9 \% ; P<$ $0.01)$, and Construction and Extraction $(5.1 \% ; P<0.01)$ occupations.

\section{DISCUSSION}

This article is the first to report national prevalence rates for three workplace psychosocial factors from the 2010 NHIS-OHS. All data presented in this study are from the public use dataset (http://www.cdc.gov/nchs/nhis/ nhis_2010_data_release.htm). 
TABLE III. Population-Based Prevalence Rates and Confidence Intervals for Psychosocial Exposures Among Working US Adults, by Industry and Occupation (National Health Interview Survey, 2010)

\begin{tabular}{|c|c|c|c|c|c|c|c|c|}
\hline & \multirow[b]{2}{*}{ Sample ${ }^{a}$} & \multirow[b]{2}{*}{ Est. population } & \multicolumn{2}{|c|}{ Job insecurity } & \multicolumn{2}{|c|}{ Work-family imbalance $^{\mathrm{b}}$} & \multicolumn{2}{|c|}{ Hostile work environment ${ }^{\mathrm{c}}$} \\
\hline & & & Exp. $^{a}$ & $\begin{array}{c}(95 \% \text { CI) } \\
(\%)\end{array}$ & Exp. $^{a}$ & $\begin{array}{l}(95 \% \text { CI) } \\
(\%)\end{array}$ & Exp. $^{a}$ & $\begin{array}{c}(95 \% \text { CI) } \\
(\%)\end{array}$ \\
\hline \multicolumn{9}{|l|}{ Industry } \\
\hline Agriculture, forestry,fishing, and hunting & 269 & 2,308 & 69 & $22.8(16.8-30.1)$ & 29 & $9.4(6.4-13.5)$ & $\dagger$ & $\dagger$ \\
\hline Mining & 75 & 721 & 23 & $34.9(23.3-48.6)$ & 14 & $20.6(11.5-34.2)$ & $\dagger$ & $\dagger$ \\
\hline Utilities & 140 & 1,447 & 23 & $17.7(11.5-26.2)$ & 21 & $14.9(9.3-23.1)$ & 11 & *6.2(3.3-11.6) \\
\hline Construction & 1,115 & 10,639 & 528 & $54.7(50.9-58.4)$ & 154 & $16.0(13.3-19.0)$ & 61 & $5.0(3.7-6.8)$ \\
\hline Manufacturing & 1,590 & 14,556 & 533 & $35.6(32.7-38.6)$ & 235 & $16.5(14.4-18.8)$ & 91 & $5.4(4.2-6.8)$ \\
\hline Wholesale trade & 396 & 3,780 & 108 & $30.4(24.9-36.5)$ & 58 & $15.0(11.4-19.6)$ & 26 & $6.5(4.2-9.9)$ \\
\hline Retail trade & 1,795 & 17,214 & 512 & $31.6(28.9-34.4)$ & 255 & $17.1(14.7-19.7)$ & 189 & $10.1(8.6-12.0)$ \\
\hline Transportation and warehousing & 714 & 6,192 & 231 & $33.5(29.2-38.0)$ & 110 & $16.7(13.7-20.2)$ & 83 & $10.2(7.9-13.0)$ \\
\hline Information & 450 & 3,854 & 172 & $45.1(39.5-50.7)$ & 67 & $18.1(14.0-23.0)$ & 28 & $7.1(4.7-10.7)$ \\
\hline Finance and insurance & 730 & 6,365 & 202 & $27.3(23.7-31.3)$ & 102 & $15.3(12.2-18.9)$ & 44 & $5.1(3.7-7.1)$ \\
\hline Real Estate and rental and leasing & 344 & 2,896 & 99 & $29.4(23.5-36.1)$ & 45 & $15.9(11.1-22.1)$ & 26 & $6.8(4.4-10.3)$ \\
\hline Professional, scientific, and technical services & 1,153 & 10,509 & 311 & $29.0(25.8-32.4)$ & 178 & $17.7(15.0-20.7)$ & 68 & $5.5(4.2-7.2)$ \\
\hline Management of companies and enterprises & $\dagger$ & $\dagger$ & $\dagger$ & $\dagger$ & $\dagger$ & $\dagger$ & $\dagger$ & $\dagger$ \\
\hline $\begin{array}{l}\text { Administrative and support and waste management } \\
\text { and remediation services }\end{array}$ & 848 & 6,895 & 328 & $39.9(35.9-44.1)$ & 130 & $16.4(13.6-19.7)$ & 66 & $6.9(5.2-9.2)$ \\
\hline Education services & 1,694 & 15,330 & 419 & $28.0(25.3-30.9)$ & 250 & $17.3(15.1-19.7)$ & 141 & $8.5(7.0-10.3)$ \\
\hline Health care and social assistance & 2,444 & 20,205 & 631 & $24.9(22.9-27.0)$ & 382 & $16.1(14.4-17.9)$ & 226 & $9.2(7.9-10.8)$ \\
\hline Arts, entertainment, and recreation & 384 & 3,420 & 117 & $32.1(26.5-38.2)$ & 48 & $13.6(9.7-18.6)$ & 32 & $7.5(5.0-11.0)$ \\
\hline Accommodation and food services & 1,223 & 10,744 & 351 & $31.1(27.9-34.6)$ & 202 & $18.4(15.8-21.4)$ & 103 & $8.5(6.8-10.5)$ \\
\hline Other services (except public administration) & 919 & 7,791 & 280 & $30.1(26.5-33.9)$ & 109 & $12.4(9.9-15.3)$ & 50 & $6.0(4.2-8.6)$ \\
\hline Public administration & 934 & 8,018 & 189 & $19.1(16.2-22.5)$ & 145 & $15.7(13.0-18.9)$ & 148 & $16.2(13.4-19.4)$ \\
\hline \multicolumn{9}{|l|}{ Occupation } \\
\hline Management & 1,497 & 14,409 & 354 & $24.2(21.6-27.1)$ & 254 & $18.0(15.8-20.4)$ & 103 & $6.4(5.1-8.0)$ \\
\hline Business and financial operations & 821 & 7,029 & 212 & $26.7(23.3-30.5)$ & 112 & $15.1(12.4-18.3)$ & 50 & $4.9(3.6-6.7)$ \\
\hline Computer and mathematical & 471 & 4,256 & 134 & $29.1(24.2-34.7)$ & 63 & $14.0(10.6-18.2)$ & 23 & $4.4(2.8-6.9)$ \\
\hline Architecture and engineering & 305 & 3,020 & 88 & $32.2(25.9-39.2)$ & 32 & $12.6(8.5-18.2)$ & 13 & $3.9(2.2-6.9)$ \\
\hline Life, physical, and social science & 180 & 1,691 & 41 & $23.8(17.0-32.3)$ & 33 & $21.3(15.0-29.4)$ & $\dagger$ & $\dagger$ \\
\hline Community and social services & 333 & 2,782 & 99 & $28.5(23.1-34.5)$ & 53 & $15.4(11.0-21.1)$ & 56 & $15.7(11.5-21.1)$ \\
\hline Legal & 195 & 1,809 & 51 & $28.8(21.8-37.1)$ & 37 & $26.1(18.9-34.8)$ & 14 & $* 7.8(4.2-14.0)$ \\
\hline Education, training, and library & 1,125 & 10,415 & 278 & $27.9(24.7-31.3)$ & 168 & $18.1(15.4-21.2)$ & 94 & $8.7(6.8-11.0)$ \\
\hline Arts, design, entertainment, sports and media & 379 & 3,251 & 103 & $31.0(25.6-37.0)$ & 47 & $13.4(9.8-18.1)$ & 27 & $7.4(4.9-11.0)$ \\
\hline Healthcare practitioners and technical & 855 & 7,285 & 171 & $18.0(15.4-21.0)$ & 159 & $19.3(16.4-22.7)$ & 79 & $8.5(6.6-10.8)$ \\
\hline Healthcare support & 485 & 3,824 & 140 & $29.8(25.1-35.0)$ & 77 & $15.3(11.6-19.9)$ & 53 & $11.1(8.1-15.0)$ \\
\hline Protective service & 358 & 3,022 & 78 & $21.4(17.0-26.6)$ & 73 & $20.9(16.2-26.6)$ & 85 & $24.6(19.6-30.5)$ \\
\hline Food preparation and serving related & 997 & 8,802 & 286 & $30.6(26.9-34.5)$ & 150 & $18.2(15.3-21.5)$ & 84 & $7.8(6.0-10.0)$ \\
\hline Building and grounds cleaning and maintenance & 767 & 6,023 & 303 & $40.8(36.4-45.3)$ & 113 & $14.4(11.6-17.8)$ & 43 & $5.7(3.9-8.3)$ \\
\hline Personal care and service & 672 & 5,734 & 200 & $28.4(24.3-32.9)$ & 81 & $13.7(10.5-17.8)$ & 42 & $7.7(5.1-11.4)$ \\
\hline Sales and related & 1,743 & 16,176, & 508 & $31.3(28.6-34.0)$ & 254 & $17.1(14.8-19.7)$ & 151 & $8.5(7.1-10.2)$ \\
\hline Office and administrative support & 2,400 & 20,497 & 676 & $30.9(28.5-33.3)$ & 304 & $13.7(12.0-15.6)$ & 223 & $9.3(7.9-10.8)$ \\
\hline Farming, fishing, and forestry & 135 & 1,048 & 43 & $34.5(24.6-45.9)$ & 16 & $9.5(5.2-16.6)$ & $\dagger$ & $\dagger$ \\
\hline Construction and extraction & 906 & 8,707 & 444 & $55.7(51.3-60.0)$ & 121 & $15.5(12.7-18.7)$ & 52 & $5.1(3.6-7.0)$ \\
\hline Installation, maintenance, and repair & 564 & 5,282 & 191 & $36.4(31.4-41.7)$ & 82 & $14.9(11.8-18.7)$ & 45 & $7.7(5.5-10.7)$ \\
\hline Production & 1,053 & 9,136 & 390 & $39.8(36.1-43.7)$ & 148 & $15.4(12.8-18.3)$ & 69 & $6.1(4.7-8.0)$ \\
\hline Transportation and material moving & 978 & 8,684 & 337 & $37.9(34.1-41.8)$ & 160 & $17.9(15.2-20.9)$ & 82 & $8.0(6.3-10.0)$ \\
\hline
\end{tabular}

Est., estimated; Exp., exposed; $\mathrm{Cl}$, confidence interval.

All estimates weighted unless otherwise noted.

aUnweighted.

${ }^{\mathrm{b}}$ Includes currently employed adults.

${ }^{\mathrm{c}}$ Includes currently employed adults, and adults not currently employed but employed at some time in the past 12 months.

* Relative standard error (RSE) $>30 \% \geq 50 \%$ are noted in the tables as they do not meet the NCHS standards of reliability/precision.

${ }^{\dagger}$ Estimates with a relative standard error $>50 \%$ or based on cell sizes $\leq 10$ are not shown as they do not meet standards of reliability/precision. 


\section{Job Insecurity}

The concept of job insecurity can refer to either perceived job insecurity or an objective measure of job loss or unemployment. The question on job insecurity in the NHIS-OHS measures perceived job insecurity. Perceived job insecurity has been measured in a number of different ways in the literature, making comparisons difficult to interpret. For example, the Quality of Worklife Survey (QWL) included in the General Social Survey [Grosch et al., 2006; Waters et al., 2007, 2011] asks respondents to rate how true the statement "The job security is good" is on a 4-point scale, while the NHIS-OHS asks respondents whether they are worried about becoming unemployed on a 4-point scale. In contrast with our findings of an overall prevalence rate of job insecurity of $31.7 \%$ in the 2010 NHIS-OHS, combined data from the QWL for 2002, and 2006 (QWL, http://www.norc.org/GSS+WebWebsite/) showed a prevalence rate of $14.8 \%$ (95\% CI 12.93-15.23) for those responding that their job security was not "good." Our prevalence rate is more than double, and may be due in part to recent worsening in economic conditions, as well as due to differences in question wording. We found especially high prevalence rates of job insecurity in workers employed in the Construction industry and Construction and Extraction occupations, but we also found relatively high prevalence rates in certain Service subsectors (e.g., Information Services).

\section{Work-Family Imbalance}

The overall prevalence rate of work-family imbalance was $16.3 \%$ in our study, which is much less than that found in the QWL (2002 and 2006 combined) using different questions to measure this construct. The NHIS-OHS question asked respondents to agree or disagree with the statement - "It is easy for me to combine work and family responsibilities." In contrast, the QWL asks the respondents two questions "how often do the demands of your job interfere with family life?" and "how often do the demands of your family interfere with your work on the job?" Responses of often and sometimes were coded as having work-family imbalance. The weighted prevalence rate for the first question was $40.7 \%$, and for the second question, $29.5 \%$. Work-family imbalance represents a multidimensional construct that is influenced by job characteristics, family characteristics, and spillover between work and family [Keene and Quadagno, 2004]. As such, interpretation based upon one question may be difficult. The 2010 NHIS-OHS shows that work-family imbalance varies by industry and occupation group, with a higher prevalence rate of workers indicating imbalance in Management of Companies and Enterprises (based on small numbers), Mining industries, and in Legal occupations.

\section{Hostile Work Environment}

Researchers have found that poor psychosocial work environments (e.g., high work pressure, autocratic management style, role conflict) may create and sustain conditions that are conducive to bullying [Bowling and Beehr, 2006; Agervold, 2009]. We found an overall prevalence rate of $7.8 \%$ for having been threatened, bullied, or harassed on the job during the previous 12 months. However, based on a probability sample of American workers, Schat et al. [2006] reported that $13 \%$ of the workforce experienced workplace psychological aggression on a weekly basis. We combined two questions in the 2002, and 2006 QWL that measure experiencing sexual harassment or other harassment or threats while on the job in the last 12 months, and found a prevalence rate of $11.3 \%$. Perhaps recent prevention programs and interventions to reduce bullying have resulted in the lower prevalence found in our study. We found a higher prevalence rate among government workers compared to other workers in the 2010 NHIS-OHS. This needs further exploration. It may be a paradoxical effect of greater job security among public workers, allowing some workers to express more dysfunctional coping mechanisms. It would also be worthwhile to explore whether the bullying comes from co-workers or from interactions with the public.

\section{Methodological Considerations}

We defined psychosocial factors as the quality or experience of various social exposures. Psychosocial factors can be examined in several ways; these factors can be seen as measures of the social environment (risk factors), but also as outcomes resulting from the organization of work and factors outside of the work environment. Psychosocial factors inside the workplace may interact with each other and result in synergistic effects associated with adverse health outcomes. Tetrachoric correlations between these three psychosocial exposures are shown in Appendix A. Correlations between exposures were relatively weak $(r<0.239)$.

Psychosocial factors are difficult to study for several reasons. There is not a standardized definition used by all researchers, and measuring these factors can be difficult. Due to time constraints, the number of workplace psychosocial questions included in the 2010 NHIS-OHS was limited, and single questions were used to measure constructs that are often measured using multiple items or scales. Job insecurity, work-family imbalance, and hostile work environment were selected because each factor represents a serious issue facing workers today. Future studies could benefit by including additional psychosocial workplace factors (e.g., effort-reward imbalance, organizational 
justice, and job engagement) as well as by using multiple items to measure these constructs.

On the other hand, primary data collection on these factors can be expensive and time consuming and are often not included in epidemiologic studies at all. As a partial solution researchers have imputed or linked job characteristics based upon occupational title to health in national surveys or cohort data. Sources of data on job characteristics that have been used for linkage or imputation include the Quality of Employment Surveys (QES) conducted by the University of Michigan in the 1970s [Schwartz et al., 1988; Muntaner et al., 1993; Alterman et al., 1994; Landsbergis et al., 2000] and the Quality of Work life Surveys (QWL) included in the General Social Survey (GSS) conducted by the National Opinion Research Center in 2002, 2006, and 2010 [Grosch et al., 2006; Waters et al., 2007, 2011]. More recently data from the Occupation Information Network $\left(\mathrm{O}^{*} \mathrm{NET}\right)$ [Peterson et al., 2001; Hadden et al., 2004; Alterman et al., 2008; Cifuentes et al., 2010; Meyer et al., 2011] have been used for this purpose. Data from the 2010 NHIS-OHS can be used in a similar way for imputation of workplace psychosocial exposures linked by occupational title or by industry. However, there is some evidence that the use of proxies for occupational exposures in place of individual job characteristics may result in misclassification and consequent bias toward the null or finding of no effect [Meyer et al., 2011].

\section{Strengths and Limitations}

This study is subject to a number of limitations. First is the use of self-report measures that may introduce reporting bias due to memory or social desirability (e.g., a tendency to underreport the experience of bullying). Second, although the population-based sample design of the NHIS allowed us to make nationally representative estimates for many variables, small numbers of respondents with specific exposures, especially within certain demographic, industry, and occupation subgroups, made some estimates unstable. Third, the use of broad industry and occupation categories lump together workers who likely have substantially different workplace exposures.

Changes in economic, technological, legal, political, and other forces affect the demographics of the workforce (i.e., composition of the workforce with respect to gender, race, ethnicity, and age) and the nature of work. Reduced job stability and increased workload are likely to influence these workplace psychosocial factors, and emphasize the need for ongoing measurement of these factors.

Limitations aside, our study has a number of strengths. The inclusion of occupational health questions in the 2010 NHIS provided an opportunity to collect information on workplace exposures that are rarely included in national surveys. The publication of nationally representative unadjusted and adjusted prevalence rates for these psychosocial factors for multiple industries and occupations will allow researchers to use these data to impute psychosocial exposures into their data by occupation or industry title [see Alterman et al., 2008]. This is important for the occupational health literature where this exposure data is lacking. More detailed analyses of the 2010 NHIS data available in the public use dataset (http:// www.cdc.gov/nchs/nhis/nhis_2010_data_release.htm) can be used to examine associations of workplace psychosocial factors with individual health outcomes along with adjustment for health behaviors and other known risk factors.

\section{CONCLUSIONS}

The overall prevalence rate of three workplace psychosocial exposures among U.S. workers examined in the 2010 NHIS-OHS ranged from $31.7 \%$ for job insecurity to $7.8 \%$ for those reporting having been threatened, bullied, or harassed by anyone on the job (hostile work environments). We also found that each of these exposures varied greatly among different industry and occupation groups. These potentially hazardous psychosocial exposures were high within certain service subsectors (e.g., high prevalence rate of job insecurity in Information industries, and high prevalence rate of hostile work environments in Public Administration industries). These exposures have implications for worker physical and mental health and should be explored further through analyses of the public use dataset. Data provided in this article can be used for benchmarking, and to identify investigation and intervention priorities.

\section{ACKNOWLEDGMENTS}

We would like to acknowledge the input and advice from our numerous colleagues at NIOSH including many from the Division of Surveillance Hazard Evaluations and Field Studies, and the Division of Applied Research and Technology, especially Dr. Leslie MacDonald, Dr. James Grosch, and Dr. Steven Sauter. Special thanks to Dr. Marie Haring Sweeney and John Sestito, JD for their support in obtaining funding for this project. We also want to acknowledge colleagues at NCHS and BLS, and our external consultants including: Dr. Joseph Grzywacz, Dr. Carles Muntaner, Dr. Paul Landsbergis, Dr. Leslie Hammer, and Dr. Sangwoo Tak. Appreciation is also extended to Dr. Rui Shen for her analysis of the QWL. 


\section{REFERENCES}

Alterman T, Grosch J, Chen X, Chrislip D, Muntaner C, Petersen MR, Krieg E. 2008. Examining associations between job characteristics and health: Linking data from the Occupational Information Network $\left(\mathrm{O}^{*} \mathrm{NET}\right)$ to two US National Health Surveys J Occup Environ Med 50(12):1401-1413.

Agervold M. 2009. The significance of organizational factors for the incidence of bullying. Scand J Psychol 50:267-276.

Alterman T, Shekelle R, Vernon V, Burau K. 1994. Decision latitude, psychologic demand, job strain and coronary heart disease in the western electric study. Am J Epidemiol 139:620-627.

Bowling N, Beehr T. 2006. Workplace harassment from the victim's perspective: A theoretical model and meta-analysis. J Appl Psychol 91(5):998-1012.

Cifuentes M, Boyer J, Lombardi DA, Punnett L. 2010. Use of $\mathrm{O}^{*} \mathrm{NET}$ as a job exposure matrix: A literature review. Am J Ind Med 53:898-914.

Cohen RA, Makuc DM. 2008. State, regional, and national estimates of health insurance coverage for people under 65 years of age: National health Interview Survey, 2004-2006. Nat Health Stat Rep $1: 1-24$.

Day JC. Population projections of the United States by age, sex, race, and Hispanic origin: 1995 to 2050, US Bureau of the Census, current population reports Washington, DC: U.S. Government Printing Office. 1996. P 25-1130. Available from: http://www.census.gov/ prod/1/pop/p25-1130/.

Division of Health Interview Statistics. 2010. 2010 National Health Interview Survey (NHIS) public use data release: NHIS survey description. National Center for Health Statistics. Hyattsville, MD: http://ftp.cdc.gov/pub/Health_Statistics/NCHS/Dataset_Documentation/ NHIS/2010/srvydesc.pdf.

Dollard M, Skinner N, Tuckey MR, Bailey T. 2007. National surveillance of psychosocial risk factors in the workplace: An international overview. Work Stress 21(1):1-29.

Grosch JW, Caruso CC, Rosa RR, Sauter SL. 2006. Long hours of work in the US: Associations with demographic and organizational characteristics, psychosocial working conditions, and health. Am J Ind Med 49:943-952.

Hadden W, Kravitz N, Muntaner C. 2004. The dimensions of work organization using the O*NET. Soc Sci Res 33:64-78.

Karasek RA, Theorell T, Schwartz JE, Schnall PL, Pieper CF, Michela JL. 1988. Job characteristics in relation to the prevalence of myocardial infarction in the US Health Examination Survey (HES) and the Health and Nutrition Examination Survey (HANES). Am J Public Health 78(8):910-918.

Keene JR, Quadagno J. 2004. Predictors of perceived work-family balance: Gender difference or gender similarity. Sociol Perspect 47(1):1-23

Landsbergis P, Theorell T, Schwartz J, Greiner BA, Krause N. 2000 Measurement of workplace exposure variables. Occup Med 15(1): $163-188$.

Meyer JD, Cifuentes M, Warren N. 2011. Association of self-rated physical health and incident hypertension with $\mathrm{O}^{*} \mathrm{NET}$ factors: Validation using a representative national survey. J Occup Environ Med 53:139-145.

Muntaner C, Eaton WW, Garrison R. 1993. Dimensions of the psychosocial work environment in a sample of the US metropolitan population. Work Stress 7(4):351-363.

Peterson NG, Mumford MD, Borman WC, Jeanneret PR, Fleishman EA, Levin KY, Campion MA, Mayfield MS, Morgeson FP, Pearlman K, Gowing MK, Lancaster AR, Silver MB, Dye DM. 2001. Understanding work using the occupational information network $\left(\mathrm{O}^{*} \mathrm{NET}\right)$ : Implications for practice and research. Pers Psychol 54:451-492.

Pieper C, LaCroix AZ, Karasek RA. 1989. The relation of psychosocial dimensions of work with coronary heart disease risk factors: A meta-analysis of five United States databases. Am J Epidemiol 129(3):483-494.

Reed DM, LaCroix AZ, Karasek RA, Miller D, MacLean CA. 1989. Occupational strain and the incidence of coronary heart disease. Am J Epidemiol 129:495-502.

Research Triangle Institute (RTI). 2008. SUDAAN (Release 10.0) [computer software]. Research Triangle Park, NC: Research Triangle Institute.

Schat ACH, Frone M, Kelloway EK. 2006. Prevalence of workplace aggression in the US workforce: Findings from a national study. In: EK Kelloway, J Barling, JJ Hurrell, editors. Handbook of workplace violence. Thousand Oaks, CA: Sage, pp 47-90.

Schwartz Je, Pieper CF, Karasek RA. 1988. A procedure for linking psychosocial job characteristics data to health. Am J Public Health 78:904-909.

Waters TR, Dick RB, Davis-Barkley J, Krieg EF. 2007. A Crosssectional study of risk factors for musculoskeletal symptoms in the workplace using data from the general social survey (GSS). J Occup Environ Med 49(2):172-184.

Waters TR, Dick RB, Krieg EF. 2011. Trends in work-related musculoskeletal disorders: A comparison of risk factors for symptoms using quality of work life data from the 2002 and 2006 general social survey. J Occup Environ Med 53(9):1013-1024.

Appendix A. Tetrachoric Correlation Matrix of Psychosocial Exposures Among Us Working Adults (National Health Interview Survey, 2010)

\section{$(1)^{2}$}

(1) Jobinsecurity

(2) Work-family imbalance

(3) Hostile work environment

1.000

0.180

0.212
(2) ${ }^{\mathrm{a}}$

Tetrachoric correlation coefficients do not account for complex sampling design.

${ }^{a}$ Estimates in column are for currently employed adults. 